\title{
La gobernabilidad metropolitana: el caso de Barcelona
}

\section{Gabriel Coloméy M ariona Tomás Fornes*}

\section{Introducción}

El caso de Barcelona es un ejemplo de las dificultades para gobernar las áreas metropolitanas y nos demuestra, una vez más, la diferencia entre la ciudad real (la que se extiende más allá de los límites administrativos) y la ciudad administrativa (en este caso los 97,6 km² del municipio barcelonés, $90 \mathrm{~km}^{2}$ reales sin el parque de Collserola). Para entenderlo, es necesario recordar brevemente las distintas fases de tranfformación y crecimiento que ha sufrido la ciudad. $H$ ay tres períodos clave para la historia de Barcelona: la ciudad medieval, ciudad amurallada, comercial y artesanal, da paso a la ciudad industrial burguesa que rompe el corsé de sus murallas para crecer hacia el llano y romper con la sospecha sobre su lealtad tras la Guerra de Sucesión. Es el período de la revolución industrial, la expansión de la ciudad, el Plan Cerdà, la ubicación de industrias, la inmigración. En definitiva, la ciudad inacabada que sobrevivirá a duras penas los años de expolio del franquismo. Finalmente, la ciudad democrática, olímpica y terciaria, en la que se produce el cambio de la industria por el sector servicios, aumenta la importancia de las dinámicas económicas internacionales y entra en juego la visión metropolitana como elemento de competitividad. La ciudad del siglo XXI, transformada en ciudad del conocimiento.

\section{Un poco de historia...}

D urante el siglo xx la ciudad de Barcelona tuvo distintos estatutos y planes para ordenar el territorio municipal y también metropolitano. Sin embargo, el contexto político e histórico dificultó su aplicación, y los cambios introducidos por el Estatuto M unicipal de 1924 o la ley municipal catalana de 1934, por ejemplo, no tuvieron continuidad.

En los años del desarrollismo de la dictadura franquista, la ciudad experimentó un fuerte crecimiento económico y demográfico, en su interior y hacia la periferia. Este hecho manifestó la necesidad de disponer de algún instrumento de planificación más adecuado para gestionar una ciudad con nuevos problemas (pobreza, barraquismo, aumento de la movilidad) agravados por la especulación económica y las «alcaldadas». Así, en 1960 Barcelona obtuvo un régimen especial orgánico y económico (conocido como Carta M unicipal), gracias al cual se creó una Comisión de Gobierno con la presencia de técnicos y también se reformaron las finanzas municipales (creación de impuestos propios). Anteriormente se habían aprobado el Plan Comarcal (1953) y el Plan Provincial (1959), con el objetivo de regular el crecimiento del área de influencia de la ciudad, que incluía 27 municipios (Ló PEZ, 2002).

El año 1964, se encargó a la Comisión de U rbanismo y Servicios Comunes de Barcelona y 0 tros M unicipios (substituta de la antigua Comisión de U rbanismo) que revisara el plan de 1953, considerado obsoleto. D os años después se presentó el Plan Director del Área M etropolitana de Barcelona, que representó un cambio de perspectiva ya que incluía 163 municipios y más de 3.000 km². En 1969 se encargó la segunda fase de revisión del Plan, presentada públicamente en 1974 como el anteproyecto Plan General de O rdenación U rbana y Territorial de la Comarca de Barcelona. Este mismo año se creó la Entidad M unicipal Me tropolitana de Barcelona1, aunque sólo incluía la ciudad y 26 municipios del alrededor con una superficie de 476 km². 
Este organismo pasó a llamarse Corporación M etropolitana de Barcelona (CM B) y estaba dotado de un Consejo M etropolitano con importantes recursos económicos (provenientes de los municipios miembros) y competencias en materia de urbanismo, transporte público, abastecimiento de agua y su depuración, tratamiento de residuos. U na de sus mayores tareas era implementar el Plan General M etropolitano (PGM), aprobado definitivamente en 1976. En los años 80, la CM B aumentó su presupuesto, gracias a fondos autonómicos (en concepto del Plan de Saneamiento) y a fondos estatales (Fondo $\mathrm{N}$ acional de Cooperación M unicipal). Durante este período, la CM B ejecutó distintos planes (saneamiento, ordenación de Collserola, costas) (ARTAL, 2002).

Sin embargo, la existencia y fortalecimiento de la CM B no estaba exenta de problemas políticos, principalmente de dos tipos. En primer lugar, existían tensiones entre los municipios pertene cientes a la Corporación a causa de la política del alcalde M aragall y la supremacía de Barcelona. Se trataba de un conflicto entre los municipios gobernados por el PSUC (luego IC) y los gobernados por el PSC (que lideraban Barcelona y otros municipios grandes). Por un lado, los comunistas acusaban al alcalde de Barcelona de actuar con prepotencia, mientras que, por el otro, al gunos de ellos no créan en el proyecto dela CM B (URíA, 1997).

No obstante, el mayor peligro de la CM B era la Generalitat de Cataluña, gobernada por los nacionalistas catalanes (CiU ). Sus dirigentes, con Jordi Pujol a la cabeza, veían con recelo el poder metropolitano (recordemos que la CM B agrupaba a municipios de gobiernos de izquierdas y representaba más de la mitad de la población catalana). Proclamándose antifranquista y considerando necesaria una reforma en el marco de la democracia, el Parlamento catalán aprovechó su competencia para legislar sobre el gobierno local y la mayoría absoluta de CiU para aprobar en 1987 las Leyes de O rganización Territorial de Cataluña2 (LOT). La abrogación de la Ley de 1974 se enmarcaba en una reforma territorial que recuperaba la figura de las comarcas, y supuso la abolición de la CM B3, de manera similar a la del Greater London Council por el Gobierno Thatcher un año antes4 (CARRERAS, 2002).

Para cubrir los servicios del área metropolitana se crearon dos nuevas entidades de carácter sectorial: la Entidad M etropolitana del Transporte (EM T) y la Entidad M etropolitana de Servicios H idráulicos y del Tratamiento de Residuos, conocida también como Entidad M etropolitana del M edio Ambiente (EMA). La primera incluye 18 municipios y se encarga del transporte público para viajeros, mientras que la segunda agrupa 33 municipios y se ocupa de abastecer y depurar el agua, tratar los residuos, etc. Las competencias en materia de urbanismo de la CM B fueron transferidas a la Administración de la G eneralitat y, en menor medida, a los Ayuntamientos. Además, la mayor parte de los municipios que pertenecían a la desparecida CM B se inte graron de manera voluntaria en la M ancomunidad de M unicipios del Área M etropolitana (M M AM B), con la finalidad de mantener los servicios comunes y elaborar proyectos de desarrollo conjunto (M M AM B, 1995).

Las tres entidades comparten su sede y su imagen corporativa, y de ellas dependen distintos organismos autónomos, consorciosy empresas metropolitanas. A esta diversidad institucional se le añade la Autoridad del Transporte M etropolitano (AT M ), que es un consorcio para la coordinación del sistema metropolitano del transporte público, constituido por la G eneral itat de Cataluña, el Ayuntamiento de Barcelona y la Entidad M etropolitana del Transporte. L'ATM ha impulsado la integración tarifaria en la Región M etropolitana, que incluye 7 comarcas, 164 municipios, más de 3.000 km² y una población de 4,5 millones de habitantes.

Una de las consecuencias principales de la fragmentación de la gestión metropolitana es la ausencia de visibilidad, lo que ge nera confusión entre los ciudadanos. En efecto, con la variedad de organismos, los ciudadanos suelen tener dificultades para identificar qué institución les representa o qué estructura les presta los servicios. Además, los miembros de los consejos de las diversas entidades son elegidos de manera indirecta, hecho que no favorece el conocimiento de los representantes y cuestiona su legitimidad. 0 tro efecto de esta multiplicidad de estructuras es la dificultad de coordinación en la aplicación de políticas, sobre todo si pensamos que, además de las entidades comentadas y los municipios del área, existe un segundo nivel de gobierno local (provincia de Barcelona6) y las comarcas7, además de la G eneralitat, el Estado, la Unión Europea y múltiples actores sociales y privados. Como veremos en el siguiente punto, un instrumento de coordinación han sido los planes estratégicos.

\section{El impulso de los Juegos O límpicos y la reconversión del área metropolitana}

Paralelamente al debate metropolitano, el área sufrió importantes cambios en los años 80 y principios de los 90, en parte a causa de la nominación olímpica, pero también a causa de las tendencias económicas y demográficas propias de las grandes ciudades.

En primer lugar, la nominación olímpica de 1992 supuso una oportunidad excelente para el desarrollo de la ciudad, especialmente en lo que se refiere a inversiones, infraestructuras y a promoción internacional o city-marketing. Los JJ0 0 renovaron 
la imagen de la ciudad, la pusieron en el mapa y la convirtieron en un escaparate para el turismo mundial. Simultáneamente, desde el Ayuntamiento se hizo un esfuerzo para promocionar Barcelona a través de las redes de ciudades, como Eurocities 0 C-6 (BORJA; DE FORN, 1990; M ORATA, 1997).

$M$ ás allá del filón olímpico, sin embargo, hubo detrás una filosofía muy clara del Ayuntamiento, un proyecto de ciudad que culminó en el denominado «modelo Barcelona». No es el objetivo de este artículo describir las características (económicas, políticas, urbanísticas, arquitectónicas) del modelo, ya que ha sido ampliamente descrito8. Aquí sólo describiremos sus aspectos fundamentales.

D esde el punto de vista urbanístico, se combinó una arquitectura «de acupuntura» con grandes actuaciones. En otras palabras, la ciudad recuperó numerosos espacios públicos que dignificaron los barrios y a la vez desarrolló obras que cambiaron la fisonomía de la ciudad (la fachada marítima y el barrio de la Villa O límpica, una nueva red de conectividad con los cinturones de ronda y túneles, las cuatro áreas olímpicas y las áreas de nueva centralidad, los equipamientos culturales) (Bo RJA, 1995).

Desde el punto de vista organizativo, destacan la descentralización de los distritos y la modernización de la gestión municipal. Las reformas, comprendidas entre 1979 y 1991, tenían un doble objetivo. Por un lado, acercar la administración al ciudadano y mejorar la relación entre gobernantes y gobernados me diante distintos instrumentos de participación ciudadana. Por otro, racionalizar la administración y mejorar el nivel de eficiencia (Ajuntament de Barcelona, 1999a).

D esde la perspectiva de la coordinación entre los distintos actores, el instrumento para articular el proyecto fueron los planes estratégicos, en un momento en que aún no era habitual en los ayuntamientos españoles. Aprovechando el impulso de los JJ00, se intentó juntar todos los actores, privados y públicos para pactar un documento que fuera la guía para el desarrollo de la ciudad, fomentando la cooperación público-privada y la participación ciudadana. En la práctica, el Plan Estratégico actúa como unificador de esfuerzos y dinamizador de los temas claves de la ciudad. En 1988 se constituyó la asociación promotora del | Plan Estratégico, aprobado en 1990. Luego le sucedieron el II Plan (1994) y el III Plan (1999)9. Aunque estos planes estaban centrados en la ciudad, eran participados por entidades metropolitanas e incorporaban todo el área como motor de crecimiento. Como veremos en el punto siguiente, en la actualidad se ha acabado de elaborar el I Plan Estratégico M etropolitano (Asociación Plan Estratégico de Barcelona, 1999).

Simultáneamente a la transformación de la ciudad olímpica, el área metropolitana experimentó un cambio de modelo económico, caracterizado por el paso de una base económica de tipo industrial a un modelo diversificado económicamente, con pre- dominio del sector terciario. Como consecuencia de la crisis de los años 70 (que representó una cáda de la ocupación en el sector secundario) y siguiendo la tendencia de otras metrópolis europeas, Barcelona se ha convertido, como muestran los indicadores, en un centro regional de servicios 10 y de turismo11. En efecto, la actividad industrial se descentralizó en favor de la primer y segunda corona metropolitanas, mientras que las numerosas fábricas de la ciudad fueron desapareciendo o transformándose en bibliotecas, empresas, centros cívicos'12 (FerRer; N EL'LO, 1991).

0 tros rasgos de la evolución de la aglomeración metropolitana se complementan con lo anterior descrito: la desconcentración de población de los municipios y ámbitos desde Barcelona (que ha perdido habitantes) hacia la periferia; la extensión física de las ciudades y de un modelo de ciudad difusa, que implica un gran consumo de suelo y tiene al tos costes ambientales; la ampliación del modo de vida o estilo de vida urbano hacia todos los espacios; el incremento de movilidad, obligada y no obligada (por trabajo, estudio o ocio) provocada por la desconcentración y favorecida por la mejora de las comunicaciones (M M AM B, 1995).

\section{Situación actual}

Actualmente, hay dos temas fundamentales en curso: la aprobación definitiva de la Carta M unicipal y el desarrollo del Primer Plan Estratégico M etropolitano.

En primer lugar, la Carta M unicipal se concibió como un instrumento específico y necesario de la ciudad de Barcelona, re tomando la iniciativa de la Carta de 1960. Se trata de una ley especial para resolver los problemas propios de una gran ciudad, posibilidad contemplada en la Ley de Bases de Régimen Local. D e acuerdo con el principio de subsidiariedad, la Carta reclama distintas competencias autonómicas y estatales para poder asumir mayor autonomía en la gestión del urbanismo, infraestructuras, educación, servicios sociales y cultura. 0 tros ejes de la Carta son, básicamente, ampliar la participación ciudadana mediante nuevos mecanismos, reforzar los mecanismos de control de la gestión municipal y favorecer la gobernabilidad. Esto se conseguiría delimitando más claramente las competencias entre el Consejo M unicipal y los órganos ejecutivos (Alcalde y Comisión de Gobierno). Sin embargo, para lograr un consenso por parte de todas las administraciones catalanas, en la C arta no se trata la cuestión metropolitana, lo cual constituye su principal debilidad (LONG0, 1999).

El texto también establece nuevos marcos jurídicos para re gular la relación del Ayuntamiento con las otras administraciones públicas y con el sector privado. Por ejemplo, se propone la 
fórmula del consorcio para gestionar conjuntamente competencias entre el Ayuntamiento y la G eneralitat. Asimismo, se fortalecen mecanismos de intervención en materia de urbanismo, vía pública y seguridad. 0 tra novedad es la creación de la Justicia M unicipal de Barcelona para resolver de manera más rápida y ágil los casos de conflicto menor. El texto también articula el marco normativo de la propia Carta y para los posibles conflictos con el Tribunal Constitucional (en caso de leyes discordes a la autonomía local). La Carta también incluye cláusulas relativas a la fiscalidad (en relación a la participación en distintos impuestos). Finalmente, con la Carta se pretende consolidar la descentralización de la ciudad, mediante nuevas competencias y recursos para los distritos (C arta M unicipal, 1999).

El texto fue aprobado por el Ayuntamiento en 1997 y por el Parlamento catalán en 1998, pero para su aplicación es necesaria la aprobación por parte de las Cortes, aún pendiente. D e hecho, existe un pulso abierto entre representantes del Ayuntamiento barcelonés y de la Generalitat con el M inistro de Administraciones Públicas para desencallar este pacto. M ientras que desde el M inisterio se quiere impulsar una ley de grandes ciudades, desde Barcelona se reclama la aceptación de la Carta antes de que ésta quede desfasada.

En segundo lugar, desde el Ayuntamiento de Barcelona se ha decidido dar un nuevo paso y acaba de elaborarse el diseño del Primer Plan Estratégico M etropolitano13, órgano de reflexión y consulta que pretende superar el alcance municipal e integrar una visión metropolitana a 10 años vista. El proyecto implica a 35 municipios de un área de $628 \mathrm{~km}^{2}$ y tres millones de personas. 0 tros actores implicados son la Cámara de Comercio, el Consorcio de la Zona Franca, la Autoridad Portuaria, los sindicatos, las universidades, Fomento y diversas entidades bancarias, centros de investigación y asociaciones del tercer sector. D e momento, la Generalitat ha decidido no participar directamente en el proyecto.

En relación a la estructura, los órganos de gobierno son el Consejo G eneral (formado por 300 miembros) y la Comisión D elegada del Consejo (como órgano ejecutivo). Los órganos de reflexión y asesoramiento son distintas Comisiones (de Estrategia, de Prospectiva, de Enlace) y el Consejo de D esarrollo Estratégico $M$ etropolitano. Distintas reuniones han tenido lugar re gularmente, con la previsión de que un primer esbozo del Plan estuviese listo para febrero de 200314. M ientras tanto, los primeros informes de los expertos señalan que los cinco puntos críticos para garantizar el desarrollo conjunto de los municipios son: movilidad y vivienda, estrategias de impulso económico, globalización y liderazgo, capital humano, convivencia ciudadana (haciendo especial énfasis en las políticas de inmigración) (Asociación Plan Estratégico M etropolitano, 2002b).
En relación a los planes estratégicos anteriores, la participación de los otros municipios del área (miembros de la EM T, de la EM A o de la M M AM B) supone un cambio de perspectiva, nacido por el propio desarrollo de los nuevos proyectos que se sitúan a escala metropolitana. Tal y como expresa el Plan, hace falta pasar de un planeamiento estratégico a «una región metropolitana que piensa estratégicamente» (Asociación Plan Estratégico M etropolitano, 2002a: 20).

Sin embargo, 7 ciudades de la segunda corona de la región metropolitana han expresado su voluntad de tomar parte en el Plan para no sentirse excluidas, sobre todo en lo que se refiere a planificación e infraestructuras. ¿D ónde termina el área de influencia de Barcelona? ¿Son 35 municipios suficientes para abarcar la realidad metropolitana o debería incorporar los 163 municipios de la región? Y, dado que esto representaría al $75 \%$ de la población catalana, ise puede considerar Cataluña como una ciudad de ciudades? ¿0 se trata de un discurso falaz? N o son preguntas especulativas, ya que su respuesta puede marcar las tendencias de desarrollo y de crecimiento hacia una zona más limitada o más extensa. I gualmente, una determinada concepción del fenómeno metropolitano (reducido a la primera corona 0 a la segunda) puede influir en el caso que se quisiera reorganizar el ámbito metropolitano en el futuro.

\section{Retos futuros}

Las expectativas y retos del área metropolitana de Barcelona se enmarcan en un contexto marcado por unas determinadas pautas sociales y demográficas, distintos proyectos urbanísticos y cambios en el modelo económico, que intentaremos esbozar a continuación.

En primer lugar, las tendencias que anunciábamos anteriormente (pérdida de habitantes de la ciudad central hacia la periferia, consumo del suelo, aumento de la movilidad) se han consolidado en los años 90 y de hecho se han extendido hacia un territorio más amplio. Además, el encarecimiento del precio del suelo y de la vivienda (sobre todo en Barcelona pero también en la primera y segunda corona metropolitanas) y los problemas asociados a la inmigración son otros rasgos del área metropolitana barcelonesa en la actualidad (IERM B, 2000)

En segundo lugar, se están llevando a cabo importantes proyectos urbanísticos en distintos puntos del área metropolitana. Entre éstos, destaca el nuevo frente litoral junto al río Besós (extremo nordeste de Barcelona y en el municipio de Sant Adrià), que se desarrolla en el marco del Fòrum 200415. También será fundamental la construcción del tren de alta velocidad, no sólo para Barcelona (que obtendrá una nueva estación 
en la Sagrera), sino para el desarrollo de toda el área16. Además, está la ampliación del aeropuerto, del puerto y de la Zona de Actividades Logísticas y también de la Fira (en la zona del Baix Llobregat) (Ajuntament de Barcelona, 1999b; M ARSHALL, 2002) .

Finalmente, la metrópolis se enfrenta al reto de adaptación a economía del conocimiento, que se caracteriza por la creciente importancia de los flujos de información, las redes, la movilidad y las nuevas tecnologías de la información y la comunicación. Este modelo económico implica, entre otras cosas, la localización de actividades densas en conocimiento, la flexibilización de los procesos productivos y la especialización productiva (CAsTELLS, 1997-98). Según TruLLÉN, Barcelona ha asumido con éxito estos cambios y ya puede ser considerada una «ciudad del conocimiento». La tendencia es que se extienda hacia otras ciudades de su entorno con una larga tradición industrial (Sabadell, Terrassa, M ataró) (T RULLÉN, 2001).

\section{A modo de conclusión}

Como nos muestra la historia, una vez más, el cambio hacia la ciudad del conocimiento se refleja también en la transformación física de Barcelona. La reconversión del que fue el «M anchester Catalán», el Poblenou, en un distrito de actividades de nueva generación (el proyecto 22@) es el ejemplo más claro de la evolución de la ciudad, donde las antiguas chimeneas se substituyen por modernos edificios y rascacielos de cristal.

En relación a las tendencias de la aglomeración metropolitana, se está utilizando «ciudad», «área» y «región» como un único concepto, ya que hemos visto que las «fronteras» de estos niveles se difuminan, anticipando una futura integración funcional de todo el conjunto mucho más grande que el actual; lo que denominamos «la ciudad de las ciudades».

Como síntesis, ésta presenta unas características fundamentales:

a) la desconcentración de actividades y de población de los municipios y ámbitos más centrales hacia los periféricos;

b) la extensión física de las ciudades, con un gran consumo de suelo;

c) la progresiva variación de todos los espacios - incluso los libres y rurales-, a la lógica propia del «modo de vida urbano»;

d) el incremento de la movilidad provocada por la desconcentración y favorecida por la mejora de las comunicaciones:

e) la tendencia a la integración funcional entre área y región.

Estos rasgos son, en definitiva, los que han articulado el de bate del Plan Estratégico M etropolitano, cuyo objetivo es establecer los grandes proyectos de transformación de la ciudad central y del área metropolitana y responder así a los retos del siglo XXI.

Notas

\footnotetext{
* Instituto de Ciencias Políticas y Sociales (ICPS).

1 D ecreto-ley 5/1974, de 7 de agosto.

2 Eran cuatro leyes relativas a las comarcas, las diputaciones, el régimen municipal y el área metropolitana de Barcelona.

3 Se trata de la Ley 7/1987 sobre «Ley por la cual se establecen y regulan actuaciones públicas especiales en la conurbación de Barcelona y en las comarcas comprendidas dentro de su zona de influencia directa».

4 Para profundizar en el caso de Londres, B. O 'Leary (1987). «W hy was the GLC abolished?», International Journal of U rban and Regional Research, 2/11: 193-217.

5 Como contrapunto a la realidad barcelonesa, la Ley de Bases de Régimen Local re conoce el carácter unitario del hecho metropolitano. Una vez más, realidad y política siguen un camino distinto.

6 La aprobación de las LOT debería haber comportado la desaparición de las provincias y las diputaciones, ya que se crearon las comarcas como órgano supramunicipal. Sin embargo, la sentencia del Tribunal Constitucional de 1981 sólo permitía la re conversión de C ataluña en provincia única, reforma que no se ha llevado a cabo y que ha supuesto el mantenimiento de las cuatro provincias (U RíA, 1997).

70 tra muestra de la fragmentación del área metropolitana es a nivel comarcal. La división comarcal histórica contemplada en las LOT no se adaptaba a la realidad me tropolitana. Así, el territorio de la desaparecida CM B se dividió entre distintas comarcas: Barcelonés (que incluye sólo 5 municipios y que estuvo al borde de la quiebra en 1994), Baix L Lobregat, Vallès 0 ccidental y M aresme (U RíA, 1997).
}

8 O. BoH IgAS (1985). Reconstrucció de Barcelona. Barcelona: Edicions 62; J. BusQUETS (1992). Barcelona. Evolución urbanística de una ciudad compacta. M adrid: Ed. M apfre; J. BorjA (ed.) (1995). Barcelona, un modelo de transformación urbana. Q uito: PGU -LAC; Ajuntament de Barcelona (1999). Barcelona: Gobierno y Gestión de la Ciudad. Una experiencia de modernización municipal. Barcelona: Ediciones D íaz de Santos.

9 El balance después de 13 años de la introducción de los Planes Estratégicos se puede contabilizar en tres planes, 39 documentos técnicos sobre metodología y temas estratégicos, 18 jornadas técnicas con la participación de 3.500 asistentes, 17 debates sobre las actuaciones previstas, 89 reuniones del Comité Ejecutivo, 27 reuniones del Consejo $\mathrm{G}$ eneral integrado por 207 miembros. Estas tareas se han llevado a cabo gracias a un presupuesto de 6,25 millones de euros (1.040 millones de pesetas) y con una oficina de coordinación (con cuatro personas) (Asociación Plan Estratégico Metropolitano, 2002a)

10 Si miramos la evolución de la distribución del empleo por sectores de actividad, vemos el crecimiento del porcentaje de población ocupada en el sector terciario (en detrimento del sector secundario): $45,6 \%$ en $1971,58,2 \%$ en 1981 , 64,7\% en 1991 y 72\% en 1996 (Institut d'Estadística de Catal unya. Censos de població).

11 Según datos del Ayuntamiento de Barcelona, se ha duplicado la capacidad hotelera de la ciudad existente antes de los JJ00, y también se ha doblado el número de pernoctaciones (Ayuntamiento de Barcelona, Anuario Estadístico). 
12 La fase siguiente de la reconversión industrial se está llevando a cabo actualmente en el barrio industrial por excelencia, Poblenou, en el marco del proyecto 22@, como veremos más adelante.

13 Para más información, consultad el web http://www.bcn2000.es/.

14 El Plan Estatégico M etropolitano fue finalmente aprobado el 10 de marzo de 2003 por un total de 26 ayuntamientos y 16 instituciones. El documento ha definido 25 propuestas que, en la segunda fase del proyecto, que acabará a finales de año, se desarrollarán en medidas concretas.
15 El Fórum Universal de las Culturas es un evento internacional que tendrá lugar entre abril y septiembre del 2004. Se concibe como un encuentro para debatir temas como la paz mundial, la sostenibilidad medioambiental y la diversidad cultural.

16 Es interesante pensar en los efectos que tendrá el tren de alta velocidad para la articulación del área metropolitana de Barcelona con el resto de $C$ ataluña. Se prevé que gracias al nuevo tren las tres capitales provinciales (Tarragona, Girona y Lleida) queden a media hora de Barcelona. Sin duda, esto puede generar una redistribución de flujos de población y actividades (económicas, culturales).

\section{Bibliografía}

Ajuntament de Barcelona (1999a). Barcelona: G obierno y Gestión de la Ciudad. Una experiencia demodernización municipal. Barcelona: Ediciones D íaz de Santos.

Ajuntament de Barcelona (1999b). Barcelona: N uevos proyectos. Barcelona: Ajuntament de Barcelona.

Asociación Plan Estratégico de Barcelona (1999). D ocumento de Trabajo no 31: III Plan Estratégico Económico y Social de Barcelona (en la perspectiva 1999-2005).

Asociación Plan Estratégico M etropolitano (2002a). Plan Estratégico M etropolitano de Barcelona (Perspectiva 2007).

Asociación Plan Estratégico M etropolitano (2002b). D ocumento de Trabajo n-2: Tendencias y temas críticos en el área metropolitana de Barcelona .

ArTAL, F. (2002). «Articulació i desarticulació de la metrópoli, 1953-1988», L'Avenç, n- 272: 50-56.

BORJA, J.; D E FORn, M. (dirs.) (1990). Barcelona y el sistema urbano europeo. BarceIona: Ajuntament de Barcelona, Col lecció Barcelona Eurociutat, $n=1$.

BorJA, J. (ed.) (1995). Barcelona, un modelo de transformación urbana. Q uito: PGULAC.

Carreras, C. (2002). «La métropolisation de Barcelone: réalité versus politique», Annales des Ponts et Chaussés, no 102: 10-14.

Carta M unicipal de Barcelona. Boletín 0 ficial del Estado, n-28, 2 de febrero de 1999.
CAstells, M . (1997-98). La era de la información: Economía, Sociedad y Cultura. (3 vol.). M adrid: Alianza Editorial.

FerReR, A. y N el'LO, O. (1991). «Barcelona: la transformació d'una ciutat industrial», Papers. Regió Metropolitana de Barcelona, n- 3: 9-30.

Institut d'Estudis Regionalsi M etropolitans de Barcelona (IERM B) (2000). Enquesta dela Regió de Barcelona 2000. Barcelona: IERM B.

Longo, F. (1999). La Carta M unicipal. Barcelona: Aula Barcelona.

LÓ PEZ, M. (2002). «Per l'autonomia i sense democràcia. Vers una carta municipal. 1924-1960», L'Avenç, n-272: 40-48.

M ancomunidad de M unicipios del Área Metropolitana de Barcelona (M M AM B) (1995). Dinàmiques metropolitanes a l'àrea i la regió de Barcelona. Barcelona: MMAMB.

M ARSH ALL, T. (2002). «La glòria olímpica i més enllà», L'Avenç, n0 272: 58-66.

M ORATA, F. (1997). «The Euroregion and the C- 6 Network: the new politics of subnational cooperation in the West-M editerranean area», en KEATING, M. y LOUGH LIN, J. (eds.). Thepolitical economy of regionalism. London: Frank Cass: 292-305.

TruLLÉn, J. (2001). La metrópoli de Barcelona cap a l'economia del conéxement: diagnoś económica i territorial de Barcelona 2001. Barcelona: Ajuntament de Barcelona.

U RíA, L. (1997). «La batalla por el territorio», M emoria de Cataluña, no 15: 225233. 